\title{
Chiral amide derivatives of ricinoleic acid and 3-hydroxynonanoic acid synthesis and cytotoxic activity
}

\author{
Sylwia Matysiak $\mathbb{D}^{1} \cdot$ Józef Kula ${ }^{1} \cdot$ Alina Błaszczyk ${ }^{2}$
}

Received: 24 December 2018 / Accepted: 16 April 2019 / Published online: 30 April 2019

(c) The Author(s) 2019

\begin{abstract}
A series of chiral ricinoleic and 3-hydroxynonanoic acid derivatives were synthesized in this study using various chemical and biochemical procedures. An effective method for preparation of methyl esters of 3-hydroxynonanoic acid from castor oil or methyl ricinoleate by ozonolysis and oxidation was developed. Simple, fast, and efficient procedures were applied to obtain different primary and secondary, cyclic and acyclic amides, including hydroxamic acids. Among 24 synthesized derivatives of ricinoleic and 3-hydroxynonanoic acids, i.e., methyl esters, amides, and hydroxamic acids, 16 compounds were obtained and described for the first time. The synthesized compounds showed activity against the tested cancer cells, but the best cytotoxic effect was observed for hydroxamic acid derived from 3-hydroxynonanoic acid (11) against HeLa cells. In general, most of the tested compounds were more toxic against HT29 than HeLa cancer cells. The results also showed that there was no significant difference between activities of $(R)$ - and $(S)$-enantiomer of particular derivatives.
\end{abstract}

Keywords Cytotoxicity $\cdot 3$-Hydroxynonanoic acid $\cdot$ Fatty acid amides $\cdot$ MTT assay $\cdot$ Ozonolysis $\cdot$ Ricinoleic acid

\section{Introduction}

Hydroxy fatty acids occur naturally in small amounts and the only representative of this group available in sufficient quantities is $(R)$-ricinoleic acid (RA) [(R,Z)-12-hydroxyoctadec-9-enoic acid] (Salywon et al. 2005). This hydroxy fatty acid is an important feedstock commonly used in different fields of industry. The most important source of RA is castor oil, in which it represents about $80-90 \%$ of all fatty acids. It is characterized by the presence of a hydroxy group in the homoallilic position and one double bond of the cis configuration making it a great raw

Supplementary information The online version of this article (https:// doi.org/10.1007/s00044-019-02348-y) contains supplementary material, which is available to authorized users.

Sylwia Matysiak

sylwia.matysiak@edu.p.lodz.pl

1 Institute of General Food Chemistry, Faculty of Biotechnology and Food Sciences, Lodz University of Technology, 90-924 Lodz, Poland

2 Department of General Genetics, Molecular Biology and Plant Biotechnology, Laboratory of Cytogenetics, Faculty of Biology and Environmental Protection, University of Lodz, 90-237 Lodz, Poland material for chemical and biochemical syntheses. There is a lot of information about RA and its derivatives and in many publications, their great biological activity is described (Pabiś and Kula 2016). For instance, amides, esters, or glycosides exert (in vitro) a potent antiproliferative effect on tumor cell lines and also have antimicrobial activity (Narasimhan et al. 2007; D’Oca et al. 2010; Dos Santos et al. 2015; Kuppala et al. 2016).

It is worth noting that most of the published results relate to the naturally available $R$ configured RA, while there is little information about its $S$ enantiomer (Fig. 1). Limited data on this compound result from the fact that it have not been identified yet in any natural source. However, in 2014 an efficient method for the preparation of $(S)$-RA was developed (Kula et al. 2014), and then, its derivatives were obtained and described for the first time (Matysiak et al. 2017, 2018).

It is well known that both the direction of biological activity and the strength of the action of chiral compounds are largely determined by the stereochemistry of the molecule. Therefore, it can be hypothesized that the configuration of the acid moiety of hydroxy fatty acids can affect the bioactivity of their derivatives. It was confirmed by our previous study, in which different $(R)$ - and $(S)$-RA amides and their acetates were synthesized and examined in terms of anticancer and antimicrobial activity. The tested 


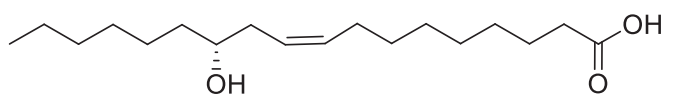

$(R)$-ricinoleic acid

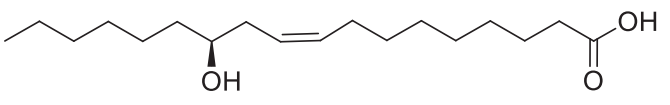

(S)-ricinoleic acid

Fig. 1 Chemical structures of enantiomeric forms of ricinoleic acid (RA)

compounds exhibited significant pharmacological potential, and what is more, differences in the activity of the opposite enantiomers were observed in some cases (Matysiak et al. 2017, 2018).

Based on this knowledge and our experience in ozonolysis (Kula and Masarweh 1998; Kula 1999; Kula et al. 2000) we decided to synthesize chiral 9-carbon skeleton derivatives of RA. Having at disposal both forms of RA methyl esters, we obtained analogous esters of $(R)$ - and $(S)$ 3-hydroxynonanoic acid by a multistage process involving ozonolysis and oxidation of castor oil and methyl $(S)$-ricinoleate, respectively.

Then, all of the obtained compounds were transformed into corresponding derivatives, i.e., amides and hydroxamic acids, and used for evaluation of their cytotoxic activity. Our attention was drawn to fatty acid amides and hydroxoamic acids, because these two groups of compounds have recently gained more and more importance in medicinal chemistry (Farrell and Merkler 2008; Pinto and Silva 2016). Amides play a crucial role in all living organisms and are commonly used in medicine as it is confirmed by the Comprehensive Medicinal Chemistry database which shows that the carboxamide group is present in more than $25 \%$ of known drugs (Montalbetti and Falque 2005).

\section{Materials and methods}

\section{Chemicals}

2-Amino-2-methyl-1-propanol, ethanolamine, pyrrolidine, hydroxylammonium chloride, Dowex 50WX8, N,O -Bis(trimethylsililyl)trifluoroacetamide+chlorotrimethylsilane (BSTFA + TMCS), and Novozyme 435 (Candida antarctica lipase) were purchased from Sigma-Aldrich. MTT [3(4,5dimethylthiazol-2-yl)-2,5-diphenyltetrazolium bromide], dimethyl sulfoxide (DMSO), penicillin-streptomycin solution stabilized and buffered saline (PBS) were purchased from Sigma Chemical Co. Foetal bovine serum (FBS), phytohemagglutinin, RPMI 1640 medium with Glutamax, Iscove's modified Dulbecco's medium, and tripsin-EDTA were supplied by Cytogen $\mathrm{GmbH}$.

Silica gel for flash chromatography (catalog no. 7024-2; J.T. Baker Co.) was purchased from Avantor Performance Materials B.V. (Deventer, Netherlands). GC-MS was performed with a Trace GC Ultra chromatograph coupled with a DSQII mass spectrometer (Thermo Scientific, Waltham, Massachusetts, USA) equipped with an Rxi-1 ms capillary column (60 m long, $0.25 \mathrm{~mm}$ inside diameter, and $0.25 \mu \mathrm{m}$ film thickness), temperature program from 220 ( $2 \mathrm{~min})$ to $330{ }^{\circ} \mathrm{C}$ at $6^{\circ} \mathrm{C} \mathrm{min}{ }^{-1}$, split $150 \mathrm{~mL} \mathrm{~min}^{-1}\left(280{ }^{\circ} \mathrm{C}\right)$, carrier gas-helium $(300 \mathrm{kPa})$, Fid $300{ }^{\circ} \mathrm{C}$, injection $0.5 \mu \mathrm{L} .{ }^{1} \mathrm{H}$ NMR $(250 \mathrm{MHz})$, and ${ }^{13} \mathrm{C}$ NMR $(62.90 \mathrm{MHz})$ were recorded using deuterochloroform solution (99.8\% $\mathrm{CDCl}_{3}$; Sigma-Aldrich), unless otherwise specified, with TMS ( $\delta=$ 0 ppm) as internal standard (Bruker DPX-250 Avance; Bruker, Hanau, Germany). ${ }^{13} \mathrm{C}$ NMR multiplicity was determined using distortionless enhancement by polarization transfer experiments. The purity of the products was confirmed by thin-layer chromatography (TLC) and ${ }^{13} \mathrm{C}$ NMR. Optical rotations were measured with an Autopol IV polarimeter (Rudolph Research, Flanders, New Jersey, USA) and IR spectra were obtained with the FT-IR spectrometer Nicolet 6700 (Thermo Scientific).

\section{Cell lines and culture conditions}

The experiments were performed with HT29 and HeLa cancer cells (human colorectal adenocarcinoma cell line and human cervical adenocarcinoma cell line, respectively). The cancer cells were obtained from the American Type Culture Collection (ATCC, Rockville, USA). The HT29 cells were cultured in RPMI 1640 medium supplemented with FBS (10\%), antibiotics: penicillin and streptomycin (1\%) and MEM non-essential amino acids solution (1\%). The HeLa cells were cultured in IMDM medium containing FBS (10\%) and antibiotics: penicillin and streptomycin (1\%), and $5 \times 10^{-5} \mathrm{M} \beta$-mercaptoethanol (1\%). All the cells were cultured at $37{ }^{\circ} \mathrm{C}$ in a humidified atmosphere of $95 \%$ air and $5 \% \mathrm{CO}_{2}$.

\section{Cytotoxicity analysis}

The effects of the tested compounds on cancer cell growth and proliferation were determined by a colorimetric MTT assay. In the living cells the water-soluble tetrazolium salt MTT (3-[4,5-dimethylthiazol-2-yl]-2,5-diphenyltetrazolium bromide; thiazolyl blue) is converted to an insoluble purple formazan by cleavage of the tetrazolium ring by mitochondrial dehydrogenase enzymes. The formazan product is solubilized with organic solvent and the absorbance is measured spectrophotometrically at $595 \mathrm{~nm}$. The cytotoxicity of 
the tested compounds is expressed as the $\mathrm{IC}_{50}$ value (concentration of the tested compound that reduces the absorbance by $50 \%$ when compared to the negative control). The assay was optimized for the cell lines and chemical compounds used in the experiments.

The cancer cells were grown for $24 \mathrm{~h}$ on $96-$ well plates at density of $6-8 \times 10^{3}$ cells/well. Then the cells were treated with the tested compounds for $72 \mathrm{~h}(2-200 \mu \mathrm{M})$. At the end of the incubation MTT dissolved in PBS was added to each plate well $(20 \mu \mathrm{L}, 5 \mathrm{mg} / \mathrm{mL})$ for $4 \mathrm{~h}$. The medium was removed and purple crystals formed in cancer cells after reduction of MTT were dissolved in DMSO (100 mL/well). Absorbance was measured with a spectrophotometer PowerWave XS (BioTek Instruments, Inc.). $\mathrm{IC}_{50}$ values were calculated by the GraphPad Prism 6.0 (GraphPad Prism Software Inc., USA). Three independent experiments were done. All the results were presented as the mean \pm SEM.

\section{Preparation of $(R)$ - and (S)-RA derivatives}

\section{Methyl esters of $(R)$ - and (S)-RA (R-1, S-1)}

Methyl $(R)$-ricinoleate $(\boldsymbol{R}-\mathbf{1})$ and methyl $(S)$-ricinoleate $(\boldsymbol{S}$ 1) were obtained according to the previously described method (Kula et al. 2014).

\section{$(R, Z)$-12-hydroxyoctadec-9-enamide $(R-2)$}

Methyl ester of $(R)$-RA $(1 \mathrm{~g}, 0.003 \mathrm{~mol})$ was added to a $2 \%$ solution of ammonia in dioxane $(25 \mathrm{ml})$ and Novozyme 435 (380 mg). The mixture was shaken at $30^{\circ} \mathrm{C}$ and $250 \mathrm{rpm}$ for $96 \mathrm{~h}$ monitoring the progress of the reaction by TLC. The enzyme was filtered off and washed by dichloromethane. Evaporation of solvent provided crude solid product which was purified by crystallization in acetone. Pure amide $\boldsymbol{R}-\mathbf{2}$ $(0.44 \mathrm{~g})$ was white solid of $98 \%$ purity $(\mathrm{GC})$ and the reaction yield was $46 \%$. $[\alpha]_{D}^{23}+2.92\left(\mathrm{c} 5.2, \mathrm{CHCl}_{3}\right), \mathrm{mp}$ 64.8-67. ${ }^{\circ} \mathrm{C}$ (acetone), lit. mp $64-65.5^{\circ} \mathrm{C}$ (Galstukhova 1960). IR ( $\mathrm{cm}^{-1}$, neat): 3353.6, 3184.0, 2922.4, 2850.8, 1658.4, 1631.8, 1469.4, 1072.9, 813.1. GC-MS (EI, 70 eV), m/z: $297\left(\mathbf{M}^{+}, 0\right), 279\left(\mathrm{M}^{+}-18,1\right), 183$ (5), 97 (10), 72 (54), 69 (15), 59 (100), 55 (70), 44 (22), 43 (35), 41 (36). ${ }^{1} \mathrm{H}$ NMR ( $\delta \mathrm{ppm}): 5.57\left(\mathrm{~m}, 3 \mathrm{H} ;-\mathrm{NH}_{2} ;-\underline{\mathrm{H}}=\right), 5.40(\mathrm{~m}, 1 \mathrm{H}$; $-\mathrm{CH}=), 3.60(\mathrm{qn} J=6.3 \mathrm{~Hz}, 1 \mathrm{H} ;-\mathrm{CHOH}), 2.21(\mathrm{~m}, 4 \mathrm{H}$; $\left.-\mathrm{C} \overline{\mathrm{H}} \mathrm{H}_{2} \mathrm{CH}=,-\mathrm{CH}_{2} \mathrm{CO}-\right), 2.04(\mathrm{~m}, 2 \mathrm{H}), 1.62$ (qn $\mathrm{J}=$ $7.3 \mathrm{~Hz}, 2 \mathrm{H}), 1.45(\mathrm{~m}, 3 \mathrm{H}), 1.30(\mathrm{~m}, 15 \mathrm{H}), 0.87(\mathrm{t} J=$ $\left.6.5 \mathrm{~Hz} ;-\mathrm{CH}_{3}\right) .{ }^{13} \mathrm{C}$ NMR: 176.08 (s; - $\left.\mathrm{CO}-\right), 133.10(\mathrm{~d}$; $-\underline{\mathrm{CH}}=), 125.24(\mathrm{~d} ;-\underline{\mathrm{CH}}=), 71.39$ (d; $-\underline{\mathrm{C}} \mathrm{HOH}-), 36.74(\mathrm{t})$, $35.82(\mathrm{t}), 35.26(\mathrm{t}), 31.75(\mathrm{t}), 29.43(\mathrm{t}), 29.27(\mathrm{t}), 29.02(\mathrm{t}$, $\left.2 \mathrm{xCH}_{2}\right), 28.94(\mathrm{t}), 27.23(\mathrm{t}), 25.63(\mathrm{t}), 25.38(\mathrm{t}), 22.53(\mathrm{t})$, $14.01\left(\mathrm{q} ;-\mathrm{CH}_{3}\right)$.

\section{$(S, Z)$-12-hydroxyoctadec-9-enamide (S-2)}

The method for its preparation was analogously as for its enantiomer $\boldsymbol{R}$-2. Process ran with $52 \%$ yield providing pure $(>98 \%, \mathrm{GC})$ amide $\mathbf{S}-\mathbf{2}$. $[\alpha]_{D}^{23}-2.95\left(\mathrm{c} 5.05, \mathrm{CHCl}_{3}\right), \mathrm{mp}$ 64.1-66 ${ }^{\circ} \mathrm{C}$ (acetone). Its IR, MS, and NMR spectra matched those determined for compound $\boldsymbol{R} \mathbf{- 2}$.

\section{$(R, Z)-N, 12$-dihydroxyoctadec-9-enamide $(R-3)$}

A solution of potassium hydroxide $(1.1 \mathrm{~g}, 0.019 \mathrm{~mol})$ in methanol $(5 \mathrm{~mL})$ and a solution of hydroxylamine hydrochloride $(0.9 \mathrm{~g}, 0.013 \mathrm{~mol})$ in methanol $(10 \mathrm{~mL})$ were obtained by heating at the boiling point of the solvent. Then, both were cooled to $30^{\circ} \mathrm{C}$ and the solution of $\mathrm{KOH}$ was added to the $\mathrm{NH}_{2} \mathrm{OH} \cdot \mathrm{HCl}$ solution. The mixture was stirred in an ice bath for $15 \mathrm{~min}$ and then the precipitated potassium chloride was filtered off. The filtrate was added to methyl ester of $(R)$-RA $(\boldsymbol{R}-1 ; 2 \mathrm{~g}, 0.006 \mathrm{~mol})$ and after thorough mixing kept at room temperature for $24 \mathrm{~h}$. Then, Dowex 50W-X8 resins were added to obtain a pH of 6 and the solution was filtered. Evaporation of methanol delivered an orange solid, therefore, crystallization in acetone was performed giving pure $(>96 \%$, GC after derivatization of the sample) hydroxamic acid $\boldsymbol{R}-\mathbf{3}(0.104 \mathrm{~g})$ with $52 \%$ yield. $[\alpha]_{D}^{23}+1.26\left(\mathrm{c} 5.0, \mathrm{CHCl}_{3}\right), \mathrm{mp} 64.9-66.3^{\circ} \mathrm{C}$ (acetone).

Derivatization with BSTFA + TMCS reagent was necessary to perform GC analysis of this compound. Therefore, $100 \mu \mathrm{L}$ of silylating reagent was added to ca. $10 \mathrm{mg}$ of sample and heated to $60^{\circ} \mathrm{C}$ for $20 \mathrm{~min}$. Then, silylated compound was dissolved in methanol in $10 \%$ concentration and subjected to GC-MS analysis.

IR ( $\mathrm{cm}^{-1}$, neat): $3403.3,3278.5,3015.5,2918.6,2847.6$, 1662.6, 1621.2, 1077.4, 855.2. GC-MS (EI, $70 \mathrm{eV}), \mathrm{m} / \mathrm{z}$ : $529\left(\mathrm{M}^{+}, 0\right), 282$ (2), 188 (14), 187 (97), 103 (31), 97 (27), 75 (20), 73 (100), 55 (32), 41 (13). ${ }^{1} \mathrm{H}$ NMR $(500 \mathrm{MHz}, \delta$ ppm): 9.39 (br.s, $1 \mathrm{H}$; -N프-), $5.52(\mathrm{~m}, 1 \mathrm{H}$; $-\mathrm{CH}=), 5.38$ (m, $1 \mathrm{H} ;--\underline{H}=), 3.62(\mathrm{qn} J=6 \mathrm{~Hz}, 1 \mathrm{H}$; $-\mathrm{CHOH}), 2.20(\mathrm{~m}, 2 \mathrm{H}$; $\left.-\mathrm{CHC}_{2}-\mathrm{CH}=\right), 2.11$ (t $\left.J=7.3 \mathrm{~Hz}, 2 \mathrm{H} ;-\mathrm{CH}_{2} \mathrm{CO}-\right), 2.03$ (m, $\left.2 \mathrm{H} ;=\mathrm{CHCH}_{2} \mathrm{CH}_{2^{-}}\right), 1.59(\mathrm{~m}, 2 \mathrm{H}), 1.46(\mathrm{~m}, 3 \mathrm{H}), 1.29(\mathrm{~m}$, $16 \mathrm{H}), 0.87\left(\mathrm{t} J=6.8 \mathrm{~Hz}, 3 \mathrm{H} ;-\mathrm{CH}_{3}\right) .{ }^{13} \mathrm{C}$ NMR $(500 \mathrm{MHz})$ : 171.69 (s; - $-\underline{C O}-), 133.24$ (d; $-\underline{-\overrightarrow{C H}}=), 125.22(\mathrm{~d} ;-\underline{\mathrm{CH}}=)$, $71.61(\mathrm{~d} ;-\mathrm{C} \overline{\mathrm{HOH}}-), 36.68(\mathrm{t}), 35.23(\mathrm{t}), 32.84(\mathrm{t}), 31.81(\mathrm{t})$, $29.36(t), 29.33(t), 28.86(t), 28.84(t), 28.82(t), 27.18(t)$, $25.69(\mathrm{t}), 25.28(\mathrm{t}), 22.60(\mathrm{t}), 14.07\left(\mathrm{q} ;-\underline{-} \mathrm{CH}_{3}\right)$.

\section{$(S, Z)-N, 12$-dihydroxyoctadec-9-enamide (S-3)}

The reaction was carried out in the same way as for compound $\boldsymbol{R}-3$. Pure $(>98 \%$, GC after derivatization of the sample) hydroxamic acid $(\boldsymbol{S}-\mathbf{3})$ was obtained with $40 \%$ yield. $[\alpha]_{D}^{23}-1.38$ (c $\left.5.25, \mathrm{CHCl}_{3}\right), \mathrm{mp} \quad 64.6-65.7^{\circ} \mathrm{C}$ 
(acetone). Conducting the $\mathrm{GC}$ analysis required a derivatisation of the sample which was done analogously as for compound $\boldsymbol{R}$-3. Its IR, MS, and NMR spectra matched those reported for enantiomer $\boldsymbol{R} \mathbf{- 3}$.

\section{$(R, Z)$-12-hydroxy- $N$-methyloctadec-9-enamide $(R-4)$}

A solution of potassium hydroxide $(1.1 \mathrm{~g}, 0.019 \mathrm{~mol})$ in methanol $(5 \mathrm{~mL})$ was added to a solution of methylamine hydrochloride $(0.9 \mathrm{~g}, 0.013 \mathrm{~mol})$ in methanol $(10 \mathrm{~mL})$ at $0{ }^{\circ} \mathrm{C}$. The mixture was stirred in an ice bath for $15 \mathrm{~min}$ and then the precipitated potassium chloride was filtered off. The filtrate was added to methyl ester of $(R)$-RA $(\boldsymbol{R}-1 ; 2 \mathrm{~g}$, $0.006 \mathrm{~mol}$ ) and after thorough mixing kept at room temperature for $24 \mathrm{~h}$. Then, Dowex 50W-X8 resins were added to obtain a pH of 6 and the solution was filtered. The solvent was evaporated and the crude product was purified on a silica gel column by using the mixture of ethyl acetate/ hexane $(40: 60, \mathrm{v} / \mathrm{v})$ as eluent. Pure ( $>99 \%, \mathrm{GC})$ compound $\boldsymbol{R}-4(1.24 \mathrm{~g})$ was obtained with $62 \%$ yield. $[\alpha]_{D}^{23}+2.35$ (c 5.05, $\left.\mathrm{CHCl}_{3}\right)$. IR $\left(\mathrm{cm}^{-1}\right.$, neat): 3297.6, 2925.2, 2854.4, 1648.1, 1560.9, 1046.9, 725.7. GC-MS (EI, $70 \mathrm{eV}), \mathrm{m} / \mathrm{z}$ : $311\left(\mathrm{M}^{+}, 0\right), 293\left(\mathrm{M}^{+}-18,1\right), 97$ (8), 86 (53), 73 (100), 69 (12), 58 (36), 55 (56), 43 (21), 41 (23). ${ }^{1} \mathrm{H}$ NMR ( $\delta$ ppm): $5.54(\mathrm{~m}, 2 \mathrm{H} ;-\mathrm{C} \underline{\mathrm{H}}=,-\mathrm{N} \underline{\mathrm{H}}-), 5.38(\mathrm{~m}, 1 \mathrm{H} ;-\mathrm{C} \underline{\mathrm{H}}=), 3.60(\mathrm{qn}$ $J=5.9 \mathrm{~Hz}, \quad 1 \mathrm{H} ; \quad-\mathrm{CHOH}), \quad 2.79 \quad(\mathrm{~d} J=4.8 \mathrm{~Hz}, \quad 3 \mathrm{H}$; $\left.-\mathrm{NHCH}_{3}\right), 2.18(\mathrm{~m}, 4 \overline{\mathrm{H}}), 2.04$ (m, 2H), 1.35 (br.m, 18H), $0.87\left(\overrightarrow{\mathrm{t} J}=6.5 \mathrm{~Hz}, 3 \mathrm{H} ;-\mathrm{CH}_{3}\right) .{ }^{13} \mathrm{C} \mathrm{NMR}: 173.92$ (s; - $\underline{\mathrm{CO}}$ ), 132.78 (d; $-\underline{\mathrm{C}} \mathrm{H}=), 125.24$ (d; $-\underline{\mathrm{C} H}=), 71.26$ (d; $-\underline{\mathrm{C}} \mathrm{HOH}-$ ), $36.66(t), 36.36(t), 35.19(t), 31.67(t), 29.36(t), 29.19(t)$, $29.06(\mathrm{t}), 29.01(\mathrm{t}), 28.89(\mathrm{t}), 27.15(\mathrm{t}), 26.01\left(\mathrm{q} ;-\mathrm{NHCH}_{3}\right)$, $25.58(\mathrm{t}), 25.55(\mathrm{t}), 22.44(\mathrm{t}), 13.90\left(\mathrm{q} ;-\mathrm{CH}_{3}\right)$.

\section{$(S, Z)$-12-hydroxy-N-methyloctadec-9-enamide (S-4)}

Reaction was carried out analogously as for compound ( $\boldsymbol{R}$ 4) starting from methyl $(S)$-ricinoleate $(\boldsymbol{S}$-1) as a substrate. Purification on column chromatography delivered compound $S$-4 with $>99 \%$ purity (GC) and $55 \%$ yield. $[\alpha]_{D}^{23}-2.31\left(\mathrm{c} 5.1, \mathrm{CHCl}_{3}\right)$. IR, MS, and NMR spectra were in accordance to compound $\boldsymbol{R} \mathbf{- 4}$.

\section{Preparation of dimethyl acetals of $(R)$ - and (S)-3- hydroxynonanal}

\section{Dimethyl acetal of $(R)-3$-hydroxynonanal $(R-5)$}

Reaction was carried out according to the previously described method (Kula et al. 2000). Ozonation conditions: $160 \mathrm{~g}$ of ozone in $\mathrm{Nm}^{3}$, gas flow $0.4 \mathrm{~g} / \mathrm{min}$, time $5 \mathrm{~h}$. Pure acetal $\boldsymbol{R}-\mathbf{5}(>96 \%, \mathrm{GC})$ was isolated by vacuum distillation with $74 \%$ yield. Bp $96-104{ }^{\circ} \mathrm{C} / 0.8$ torr, lit. bp $90-92{ }^{\circ} \mathrm{C} / 0.7$ torr (Kula et al. 2000); $[\alpha]_{D}^{23}+12.2$ (c 5.16, $\mathrm{CHCl}_{3}$ ); lit.
$[\alpha]_{D}^{21}-13.7$ (c 0.9, MeOH) (Kula et al. 2000). IR $\left(\mathrm{cm}^{-1}\right.$, neat): 3432.7, 2927.2, 2856.1, 1457.5, 1192.4, 1120.4, 1054.4, 820.7. GC-MS (EI, $70 \mathrm{eV}), \mathrm{m} / z: 204\left(\mathrm{M}^{+}, 0\right), 173$ (2), 119 (5), 97 (3), 87 (33), 75 (100), 59 (36), 58 (13), 55 (7), 43 (4). The enantiomeric purity of the product was $>99 \% e e$, which was determined by chiral GC analysis described elsewhere (Kula et al. 2014).

\section{Dimethyl acetal of (S)-3-hydroxynonanal (S-5)}

Methyl $(S)$-ricinoleate $(\boldsymbol{S}-\mathbf{1})(71.5 \mathrm{~g}, 0.23 \mathrm{~mol})$ was dissolved in methanol $(250 \mathrm{~mL})$ and the mixture was cooled to $-10^{\circ} \mathrm{C}$. Oxygen containing $160 \mathrm{~g}$ of ozone in $\mathrm{Nm}^{3}$ at the gas flow $0.4 \mathrm{~g} / \mathrm{min}$ was passed through the solution until a positive test with potassium iodide (ozonation time: $2.5 \mathrm{~h}$ ). When the reaction was completed, the peroxides were reduced by addition of dimethyl sulphide (DMS, $50 \mathrm{~g}$, $0.8 \mathrm{~mol})$. DMS was added with stirring at temperature below $0{ }^{\circ} \mathrm{C}$ for $1.5 \mathrm{~h}$. Then, the reaction mixture was left overnight at room temperature until the total decomposition of peroxides, which was controlled by the potassium iodide test. Next, the solution containing (S)-3-hydroxynonanal as intermediate was cooled to $-5{ }^{\circ} \mathrm{C}$ and $35 \% \mathrm{HCl}(4.5 \mathrm{~mL})$ in methanol $(75 \mathrm{~mL})$ was dropped carefully during intensive stirring, maintaining the temperature below $0{ }^{\circ} \mathrm{C}$. Stirring was continued for $2 \mathrm{~h}$ at room temperature and left overnight. In the next step, $\mathrm{KOH}(40 \mathrm{~g}, 0.07 \mathrm{~mol})$ in water $(50 \mathrm{~mL})$ was added at $0-5{ }^{\circ} \mathrm{C}$. Excess of DMS with a little of methanol (entirely $100 \mathrm{~mL}$ ) were distilled off and the residue was refluxed for $3 \mathrm{~h}$. In this way, present in the mixture glycerides were converted into water-soluble potassium salts easy to remove during extraction. After vacuum evaporation of methanol and addition of water $(100 \mathrm{~mL})$, the extraction with diethyl ether $(3 \times 100 \mathrm{~mL})$ was carried out. The ether solution was washed neutral with water and dried over anhydrous $\mathrm{MgSO}_{4}$. Then, the solvent was evaporated and crude product was isolated by vacuum distillation giving pure ( $>97 \%$, GC) acetal $S$-5 $(32 \mathrm{~g}, 68 \%$ yield). Bp $89-88^{\circ} \mathrm{C} / 0.2$ torr; $[\alpha]_{D}^{23}-11.95$ (c $5.08, \mathrm{CHCl}_{3}$ ). IR, MS, and NMR spectra were in accordance with compound $\boldsymbol{R} \mathbf{- 5}$. The enantiomeric purity of the product was $94.7 \% e e$, which was determined by chiral GC analysis described elsewhere (Kula et al. 2014).

\section{Preparation of $(R)$ - and (S)-3-hydroxynonanoic acid derivatives}

\section{Methyl ester of $(R)$-3-hydroxynonanoic acid $(R-6)$}

Oxidation of $(R)$-3-hydroxynonanal dimethyl acetal $(\boldsymbol{R}-\mathbf{5})$ to corresponding methyl ester ( $\boldsymbol{R}-\mathbf{6})$ was carried out similarly as published by Takeda et al. (Takeda et al. 1997). Compound $\boldsymbol{R}-5$ (20 g, $0.098 \mathrm{~mol}$ ) was dissolved in methanol 
(200 mL) and cooled to $0{ }^{\circ} \mathrm{C}$. In the next step, $35 \% \mathrm{HCl}$ $(15.5 \mathrm{~g}, 0.15 \mathrm{~mol})$ and then $30 \% \mathrm{H}_{2} \mathrm{O}_{2}(17 \mathrm{~g}, 0.15 \mathrm{~mol})$ were dropped carefully during intensive stirring maintaining temperature below $5{ }^{\circ} \mathrm{C}$. The stirring was continued for an additional $30 \mathrm{~min}$ in an ice bath. Then, the solution was heated to $40{ }^{\circ} \mathrm{C}$ and stirred in this temperature for $3 \mathrm{~h}$. About $100 \mathrm{~mL}$ of methanol was evaporated and after addition of water $(80 \mathrm{~mL})$ the extraction with diethyl ether was performed $(3 \times 100 \mathrm{~mL})$. The organic layer was washed with aqueous sodium sulfide $(80 \mathrm{~mL})$, then with water $(80 \mathrm{~mL})$ and dried over anhydrous $\mathrm{MgSO}_{4}$. The solvent was evaporated and crude product was purified on column chromatography using hexane/ethyl acetate $(80: 20 \mathrm{v} / \mathrm{v})$ as eluent to obtain pure $(>97 \%$, GC) methyl ester of $(R)-3$ hydroxynonanoic acid $(\boldsymbol{R}-\mathbf{6})\left(9.1 \mathrm{~g}, 49 \%\right.$ yield). $[\alpha]_{D}^{23}-19.3$ (c 5.10, $\mathrm{CHCl}_{3}$ ); lit. $[\alpha]_{D}^{25}-20.0$ (Odham and Samuelsen 1970), $[\alpha]_{D}^{25}-16.0$ (c 1.48, $\mathrm{CHCl}_{3}$ ) (Jiang et al. 2010). IR ( $\mathrm{cm}^{-1}$, neat): 3473.3, 2953.9, 2927.9, 1731.1, 1162, 1055.6, 864.6. GC-MS (EI, $70 \mathrm{eV}), \mathrm{m} / \mathrm{z}: 188\left(\mathrm{M}^{+}, 0\right), 170\left(\mathrm{M}^{+}-18\right.$, 1), 138 (4), 103 (100), 96 (9), 74 (36), 71 (38), 61 (16), 55 (21), 43 (43), 41 (22). ${ }^{1} \mathrm{H}$ NMR ( $\left.\delta \mathrm{ppm}\right): 3.95(\mathrm{~m}, 1 \mathrm{H}$; $-\mathrm{C} \underline{\mathrm{HOH}}$ ), 3.66 (s, 3H; - $\left.\mathrm{OCH}_{3}\right), 2.47$ (dd $J=16.3 \mathrm{~Hz}, J=$ $3.8 \mathrm{~Hz}, 1 \mathrm{H}$; $\left.-\mathrm{CHCH}_{2} \mathrm{CO}-\right), 2.36(\mathrm{dd} J=16.3 \mathrm{~Hz}, J=$ $8.8 \mathrm{~Hz}, 1 \mathrm{H}$; $-\mathrm{CHCH}_{2} \mathrm{CO}-$ ), 1.31 (br. $\mathrm{m}, 11 \mathrm{H}$ ), 0.83 (t $J=$ 6.6, 3H; $\left.-\mathrm{CH}_{3}\right) .{ }^{13} \mathrm{C}$ NMR: 173.34 (s; - $\underline{C O}$ ), 67.90 (d; $-\underline{\mathrm{CHOH}}), 51.57\left(\mathrm{q} ;-\mathrm{OCH}_{3}\right), 41.12(\mathrm{t}), 38.48(\mathrm{t}), 31.65(\mathrm{t})$, $29.07(\mathrm{t}), 25.33(\mathrm{t}), 22.47(\mathrm{t}), 13.92\left(\mathrm{q} ;-\mathrm{CH}_{3}\right)$.

\section{Methyl ester of (S)-3-hydroxynonanoic acid (S-6)}

The reaction was carried out in the same way as for compound $\boldsymbol{R}$-6. Pure $(97 \%, \mathrm{GC})$ methyl ester of $(S)$-3-hydroxynonanoic acid $(\boldsymbol{S}$-6) was obtained with $51 \%$ yield. $[\alpha]_{D}^{23}+18.1\left(\mathrm{c} 5.01, \mathrm{CHCl}_{3}\right)$. Its IR, MS, and NMR spectra matched those reported for enantiomer $\boldsymbol{R}-\mathbf{6}$.

\section{(R)-3-hydroxynonandamide $(R-7)$}

$25 \%$ water solution of ammonia $(8 \mathrm{~mL})$ was added to methyl ester of $(R)$-3-hydroxynonanoic acid $(\boldsymbol{R}-\mathbf{6} ; 2 \mathrm{~g}$, $0.011 \mathrm{~mol})$. The mixture was shaken at $30^{\circ} \mathrm{C}$ for $24 \mathrm{~h}$ and the progress of the reaction was monitored by TLC. The precipitated crystals were filtered off and purified by crystallization in acetone. Amide $\boldsymbol{R}-7$ (0.913 g) was white solid of $>99 \%$ purity (GC) obtained with $50 \%$ yield. $[\alpha]_{D}^{23}-26.77$ (c $0.31, \mathrm{CHCl}_{3}$ ), mp $101.8-103.7^{\circ} \mathrm{C}$ (acetone). IR $\left(\mathrm{cm}^{-1}\right.$, neat): 3349, 3176.1, 2951.8, 2921.5, 2844.4, 1631.4, 1599.4, 1095.4, 862.3. GC-MS (EI, $70 \mathrm{eV}), \mathrm{m} / \mathrm{z}: 173\left(\mathrm{M}^{+}\right.$, 0), $155\left(\mathrm{M}^{+}-18,1\right), 88$ (100), 71 (10), 59 (49), 55 (17), 45 (16), 44 (24), 43 (32), 41 (21), 39 (16). ${ }^{1} \mathrm{H}$ NMR ( 8 ppm): $5.94\left(\mathrm{~s}, 1 \mathrm{H} ;-\mathrm{NH}_{2}\right), 5.77\left(\mathrm{~s}, 1 \mathrm{H} ;-\mathrm{NH}_{2}\right), 4.00(\mathrm{~m}, 1 \mathrm{H}$; $-\mathrm{CHOH}), 3.40$ (br.s, 1H; -OH), 2.41 (dd $J=15.5 \mathrm{~Hz}, J=$ $3 \mathrm{~Hz}, 1 \mathrm{H} ;-\mathrm{CHCH}_{2} \mathrm{CO}$ ), 2.30 (dd $J=15.5 \mathrm{~Hz}, J=8.8 \mathrm{~Hz}$,
$1 \mathrm{H}$; - $\mathrm{CHCH}_{2} \mathrm{CO}$-), 1.41 (br.m, $10 \mathrm{H}$ ), 0.88 (t $J=6.6 \mathrm{~Hz}, 3 \mathrm{H}$; $\left.-\mathrm{CH}_{3}\right) .{ }^{13} \mathrm{C}$ NMR: 175.05 (s; - $\left.\mathrm{CO}-\right), 68.48$ (d; - $\left.-\mathrm{CHOH}\right)$, $42.01(\mathrm{t}), 36.87(\mathrm{t}), 31.73(\mathrm{t}), 29.15(\mathrm{t}), 25.42(\mathrm{t}), 22.55(\mathrm{t})$, $14.01\left(\mathrm{q} ;-\mathrm{CH}_{3}\right)$.

\section{(S)-3-hydroxynonandamide (S-7)}

The reaction was carried out in the same manner as for compound $\boldsymbol{R}-\mathbf{7}$. Amide $\mathbf{S - 7}$ of $>99 \%$ purity (GC) was obtained with $58 \%$ yield. $[\alpha]_{D}^{23}+26.24\left(\mathrm{c} 0.32, \mathrm{CHCl}_{3}\right)$, mp 101.4-102. ${ }^{\circ} \mathrm{C}$ (acetone). Its IR, MS, and NMR spectra matched those reported for enantiomer $\boldsymbol{R}-\mathbf{7}$.

\section{$N$-(2-hydroxyethyl)-(3R)-hydroxynonanamide $(R-8)$}

Methyl ester of (R)-3-hydroxynonanoic acid $(\boldsymbol{R}-\mathbf{6} ; 0.5 \mathrm{~g}$, $0.0027 \mathrm{~mol})$ was mixed with ethanolamine $(1 \mathrm{~g}, 0.016 \mathrm{~mol})$ and heated at $130{ }^{\circ} \mathrm{C}$ (gentle reflux) for $2 \mathrm{~h}$ by monitoring the progress of the reaction by TLC. The crude amides were purified by flash chromatography with silica gel using ethyl acetate/methanol (97:3, v/v) as eluent to deliver pure amide $\boldsymbol{R}-8$ ( $0.43 \mathrm{~g}$; $99 \%$ purity confirmed by GC) with $74 \%$ yield. Product was white solid, mp $74.3-75.9^{\circ} \mathrm{C}$ (ethyl acetate/ methanol), $79.6-80.6^{\circ} \mathrm{C}$ (acetone), $[\alpha]_{D}^{23}-9.7$ (c 5.1, $\mathrm{CHCl}_{3}$ ). IR ( $\mathrm{cm}^{-1}$, neat): 3298.6, 2920.9, 2848.5, 1643.6, 1560.7, 1081.1, 1058.4, 860.9. GC-MS (EI, $70 \mathrm{eV}), \mathrm{m} / \mathrm{z}$ : $217\left(\mathrm{M}^{+}, 1\right), 199\left(\mathrm{M}^{+}-18,1\right), 174$ (16), 139 (25), 132 (100), 114 (29), 88 (30), 62 (35), 60 (40), 55 (61), 43 (71). ${ }^{1} \mathrm{H}$ NMR ( $\left.\delta \mathrm{ppm}\right): 7.04$ (t $J=5.1 \mathrm{~Hz}, 1 \mathrm{H}$; $-\mathrm{NH}-$ ), 4.41 (br.s, $2 \mathrm{H}), 3.95(\mathrm{~m}, 1 \mathrm{H}$; $-\mathrm{CHOH}), 3.63\left(\mathrm{~m}, 2 \mathrm{H} ;-\overline{\mathrm{C}} \underline{\mathrm{H}}{ }_{2} \mathrm{OH}\right), 3.42$ (m, $1 \mathrm{H}$; - $\left.\mathrm{NHCH}_{2}-\right), 3.29\left(\mathrm{~m}, 1 \mathrm{H}\right.$; - $\left.\mathrm{NHCH}_{2}-\right), \overline{2.38}(\mathrm{dd} J=$ $\left.14.6 \mathrm{~Hz}, \quad J=2.4 \mathrm{~Hz}, 1 \mathrm{H} ;-\mathrm{CHCH}_{2} \mathrm{CO}-\right), 2.24(\mathrm{dd} J=$ $14.6 \mathrm{~Hz}, J=9.4 \mathrm{~Hz}, 1 \mathrm{H}$; $-\mathrm{CHCH}_{2} \mathrm{CO}$ ), 1.39 (br.m, $10 \mathrm{H}$ ), 0.85 (t $\left.J=6.8 \mathrm{~Hz}, 3 \mathrm{H},-\mathrm{CH}_{3}\right) .{ }^{13} \mathrm{C}$ NMR: 173.32 (s; $-\underline{\mathrm{CO}}-$ ), $68.70(\mathrm{~d},-\underline{\mathrm{CHOH}}), \quad 6 \overline{1.25}\left(\mathrm{t}, \quad-\underline{\mathrm{CH}}_{2} \mathrm{OH}\right), 43.2 \overline{7}(\mathrm{t}$, $\left.-\mathrm{CHCH}_{2} \mathrm{CO}-\right), 42.03$ (t, $\left.-\mathrm{NHCH}_{2}-\right), 37.19$ (t), 31.73 (t), $29.18(\mathrm{t}), 25.51(\mathrm{t}), 22.54(\mathrm{t}), 14.00\left(\mathrm{q},-\mathrm{CH}_{3}\right)$.

\section{$\mathrm{N}$-(2-hydroxyethyl)-(3S)-hydroxynonanamide (S-8)}

The reaction was carried out analogously to the compound $\boldsymbol{R - 8}$, however, in order to obtain pure $(>99 \%, \mathrm{GC})$ product it was necessary to perform additional purification process, i.e., crystallization in acetone. Pure amide $\boldsymbol{S - 8}$ was obtained with $39 \%$ yield, and it was a white solid. $[\alpha]_{D}^{23}+10.48$ (c 5.1, $\mathrm{CHCl}_{3}$ ); mp $79.8-80.5^{\circ} \mathrm{C}$ (acetone).

\section{$N$-(1-hydroxy-2-methylpropan-2-yl)-(3R)- hydroxynonanamide $(R-9)$}

2-amino-2-methyl-1-propanol $(6.4 \mathrm{~g}, 0.072 \mathrm{~mol})$ was added to methyl ester of $(R)$-3-hydroxynonanoic acid $(\boldsymbol{R}-\mathbf{- 6} ; 2.3 \mathrm{~g}$, $0.012 \mathrm{~mol}$ ) and the mixture was heated at $130^{\circ} \mathrm{C}$ for $2.5 \mathrm{~h}$. 
The progress of the reaction was monitored by TLC analysis. The crude product was purified by passing through a silica gel column using ethyl acetate/hexane $(50: 50, \mathrm{v} / \mathrm{v})$ as eluent. The obtained with $95 \%$ yield product $(\boldsymbol{R}-\mathbf{9}, 2.85 \mathrm{~g})$ was a light yellow dense liquid of $98 \%$ purity (GC after derivatization of the sample). $[\alpha]_{D}^{23}=-14.76$ (c 5.45, $\mathrm{CHCl}_{3}$ ). GC analysis required a previous derivatization of the sample which was done analogously as for compound R-3. IR ( $\mathrm{cm}^{-1}$, neat): 3243.2, 3074.5, 2926.6, 2856.4, 1638.9, 1553.2, 1056.8, 784.9. GC-MS (EI, $70 \mathrm{eV}$ ), $\mathrm{m} / \mathrm{z}$ : $461\left(\mathrm{M}^{+}, 0\right), 374$ (4), 286 (22), 246 (6), 187 (7), 145 (13), 144 (33), 75 (23), 73 (73), 58 (100), 55 (12). ${ }^{1} \mathrm{H}$ NMR $(\delta$ ppm): 6.65 (s, 1H; -Nㅍ-), 5.15 (br.s, $1 \mathrm{H}$; $-\mathrm{CHOH}$ ), 4.24 (br. $\mathrm{s}, 1 \mathrm{H}$; $\left.-\mathrm{CH}_{2} \mathrm{OH}\right), 3.8 \overline{3}(\mathrm{~m}, 1 \mathrm{H}$; $-\mathrm{CHOH}), 3.51 \overline{(\mathrm{d} J}=4 \mathrm{~Hz}$, $\left.1 \mathrm{H} ;-\underline{\mathrm{C}}_{2} \mathrm{OH}\right), 3.40\left(\mathrm{~d} J=4 \mathrm{~Hz}, 1 \overline{\mathrm{H}} ;-\mathrm{CH}_{2} \mathrm{OH}\right), 2.25(\mathrm{dd} J$ $\left.=5.4 \mathrm{~Hz}, J=1.1 \mathrm{~Hz}, 1 \mathrm{H} ;-\mathrm{CHCH}_{2} \mathrm{CO}\right), 2.14(\mathrm{dd} J=$ $5.3 \mathrm{~Hz}, J=3.3 \mathrm{~Hz}, 1 \mathrm{H}$; $\left.-\mathrm{CHCH}_{2} \mathrm{CO}-\right), 1.40(\mathrm{~m}, 1 \mathrm{H}), 1.32$ $(\mathrm{m}, 2 \mathrm{H}), 1.19(\mathrm{~m}, 13 \mathrm{H}), 0.88\left(\mathrm{t} J=6.8 \mathrm{~Hz}, 3 \mathrm{H},-\mathrm{CH}_{2} \mathrm{CH}_{3}\right)$. ${ }^{13} \mathrm{C}$ NMR: 173.20 (s; - $\left.\underline{\mathrm{CO}}-\right), 69.51\left(\mathrm{t},-\mathrm{C}_{2} \mathrm{OH}\right), 68.59$ (d, - $\underline{\mathrm{C}} \mathrm{HOH}), 55.55$ (s, - $\left.\underline{-}\left(\mathrm{CH}_{3}\right)_{2}-\right), 43.29$ (t, $\left.-\underline{\mathrm{CH}}_{2} \mathrm{CO}-\right), 36.89$ $(\mathrm{t}), 31.55(\mathrm{t}), 29.00(\mathrm{t}), 25.26(\mathrm{t}), 24.28\left(\mathrm{q} ;-\mathrm{C}\left(\mathrm{C}_{3}\right)_{2}-\right)$, $23.94\left(\mathrm{q} ;-\mathrm{C}\left(\mathrm{CH}_{3}\right)_{2}-\right), 22.35$ (t), $13.82\left(\mathrm{q},-\mathrm{CH}_{2} \underline{\mathrm{CH}}_{3}\right)$.

\section{$N$-(1-hydroxy-2-methylpropan-2-yl)-(3S)- hydroxynonanamide $(S-9)$}

The reaction was carried out in the same way as for compound $\boldsymbol{R}$-9. Pure $(97 \%$, GC after derivatization of the sample) amide $\boldsymbol{S - 9}$ was obtained with $87 \%$ yield. $[\alpha]_{D}^{23}+16.01$ (c 5.65, $\mathrm{CHCl}_{3}$ ). Its IR, MS, and NMR spectra matched those reported for enantiomer $\boldsymbol{R}-\mathbf{9}$.

\section{Pyrrolidinyl-(3R)-hydroxynonanamide $(R-10)$}

The mixture of methyl ester of $(R)$-3-hydroxynonanoic acid $(\boldsymbol{R}-6 ; 1.1 \mathrm{~g}, 0.006 \mathrm{~mol})$ and pyrrolidine $(2.5 \mathrm{~g}, 0.035 \mathrm{~mol})$ was heated under gentle reflux $\left(75-80^{\circ} \mathrm{C}\right)$. The lack of visible progress of the reaction on TLC was the reason for completing the process after $3 \mathrm{~h}$. Pure $(>99 \%, \mathrm{GC})$ product was obtained by purification on a silica gel column using ethyl acetate/hexane $(5: 95, \mathrm{v} / \mathrm{v})$ as eluent. The reaction yield was $67 \%$ that gave $0.89 \mathrm{~g}$ of compound $\boldsymbol{R}-10$. $[\alpha]_{D}^{23}-42.05$ (c 5.20, $\mathrm{CHCl}_{3}$ ). IR ( $\mathrm{cm}^{-1}$, neat): 3410.1, 2926.9, 2857.4, 1618.2, 1449.7, 1044.6, 857.9. GC-MS (EI, $70 \mathrm{eV}), \mathrm{m} / \mathrm{z}$ : $227\left(\mathrm{M}^{+}, 2\right), 209$ (10), 156 (7), 142 (100), 113 (60), 98 (94), 85 (15), 70 (50), 55 (50), 43 (43). ${ }^{1} \mathrm{H}$ NMR $(500 \mathrm{~Hz}, \delta$ ppm): 3.96 (m, 2H; -CHOH, -OH), 3.41 (t $J=6.8 \mathrm{~Hz}, 2 \mathrm{H}$; $\left.-\mathrm{NCH}_{2} \mathrm{CH}_{2}-\right), \quad 3.34 \quad\left(\mathrm{~m}, \quad 2 \mathrm{H} ; \quad-\mathrm{NCH}_{2} \mathrm{CH}_{2}-\right), 2.38$ (dd $J=16.3 \mathrm{~Hz}, J=2.3 \mathrm{~Hz}, 1 \mathrm{H}$; $\left.-\mathrm{CHCH}_{2} \mathrm{CO}-\right), 2.22$ (dd $J=$ $16.5 \mathrm{~Hz}, \quad J=9.5 \mathrm{~Hz}, 1 \mathrm{H} ;-\mathrm{CHCH}_{2} \mathrm{CO}-$ ), 1.91 (qn $J=$ $\left.6.6 \mathrm{~Hz}, 2 \mathrm{H} ;-\mathrm{NCH}_{2} \mathrm{CH}_{2}-\right), 1.82\left(\mathrm{~m}, 2 \mathrm{H},-\mathrm{NCH}_{2} \underline{\mathrm{CH}_{2}}\right), 1.50$ (m, $1 \mathrm{H}), 1.38(\mathrm{~m}, 2 \mathrm{H}), 1.24$ (br.m, 7H), 0.82 (t $J=6.8 \mathrm{~Hz}$,
3H, $\left.-\mathrm{CH}_{3}\right) .{ }^{13} \mathrm{C}$ NMR: 171.27 (s; -CO-), 67.83 (d; - $\left.\mathrm{CHOH}\right)$, $46.44(\mathrm{t}), 45.31(\mathrm{t}), 40.46(\mathrm{t}), 36.37(\mathrm{t}), 31.65(\mathrm{t}), 29.13(\mathrm{t})$, $25.76(\mathrm{t}), 25.36(\mathrm{t}), 24.17(\mathrm{t}), 22.44(\mathrm{t}), 13.92\left(\mathrm{q} ;-\mathrm{CH}_{3}\right)$.

\section{Pyrrolidinyl-(3S)-hydroxynonanamide (S-10)}

Amide $\boldsymbol{S - 1 0}$ was obtained in the same way as compound $\boldsymbol{R}$ 10 with $86 \%$ yield. Its purity was high $(>99 \%, \mathrm{GC})$ and the obtained IR, MS, and NMR spectra were in accordance with those for enantiomer $\boldsymbol{R}-\mathbf{1 0}$. $[\alpha]_{D}^{23}+42.30$ (c 5.30, $\mathrm{CHCl}_{3}$ ).

\section{(R)-N,3-dihydroxynonanamide $(R-11)$}

Hydroxamic acids were obtained according to methods described in literature (Hauser and Renfrow 1939; Devlin et al. 1975). A solution of potassium hydroxide $(3.6 \mathrm{~g}$, $0.064 \mathrm{~mol})$ in methanol $(10 \mathrm{~mL})$ and a solution of hydroxylamine hydrochloride $(3 \mathrm{~g}, 0.043 \mathrm{~mol})$ in methanol $(20 \mathrm{~mL})$ were obtained by heating at the boiling point of the solvent. Then, both were cooled to $30^{\circ} \mathrm{C}$ and the solution of $\mathrm{KOH}$ was added to the $\mathrm{NH}_{2} \mathrm{OH} \cdot \mathrm{HCl}$ solution. The mixture was stirred in an ice bath for $15 \mathrm{~min}$ and then the precipitated potassium chloride was filtered off. The filtrates were added to methyl ester of $(R)$-3-hydroxynonanoic acid $(\boldsymbol{R}-\mathbf{6} ; 4 \mathrm{~g}, 0.021 \mathrm{~mol})$ and after thorough mixing kept at room temperature for $24 \mathrm{~h}$. Appeared in the mixture white crystals of potassium ( $R$ )-3-hydroxynonanhydroxamate were dissolved by heating the methanol solution. Then, Dowex 50W-X8 resin was added to obtain a $\mathrm{pH}$ of 6 , next, the solution was filtered and evaporated. Crystallization in acetone was performed giving pure ( $>98 \%$, GC after derivatization of sample) hydroxamic acid $\boldsymbol{R}-\mathbf{1 1}(1.91 \mathrm{~g})$ with $47 \%$ yield. $[\alpha]_{D}^{23}-4.36$ (c $\left.2.75, \quad \mathrm{MeOH}\right)$, m.p.: $104.8-107.8^{\circ} \mathrm{C}$ (acetone). It was necessary to perform derivatization of the sample for GC analysis which was done the same way as for compound $\boldsymbol{R} \mathbf{- 3}$. IR $\left(\mathrm{cm}^{-1}\right.$, neat): 3361.1, 3294.3, 2956.2, 2922.6, 2850.9, 1647.6, 1606.4, 1081.2, 977.2. GC-MS (EI, $70 \mathrm{eV}), \mathrm{m} / z: 405\left(\mathrm{M}^{+}, 0\right), 187$ (63), 103 (19), 100 (19), 97 (8), 75 (15), 73 (100), 69 (26), 55 (39), 45 (12), 43 (11). ${ }^{1} \mathrm{H}$ NMR ( $\left.\mathrm{CD}_{3} \mathrm{OH}, \delta \mathrm{ppm}\right): 5.05$

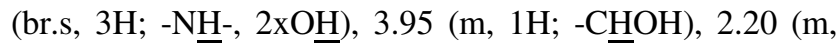
$\left.2 \mathrm{H} ;-\mathrm{CHCH}_{2} \overline{\mathrm{CO}}-\right), 1 . \overline{45}(\mathrm{~m}, 3 \mathrm{H}), 1.30(\mathrm{~m}, \overline{7} \mathrm{H}), 0.89(\mathrm{t} J=$ $\left.6.5 \mathrm{~Hz}, 3 \mathrm{H},-\mathrm{CH}_{3}\right) .{ }^{13} \mathrm{C}$ NMR $\left(\mathrm{CD}_{3} \mathrm{OH}\right): 170.96$ (s, $\left.-\underline{\mathrm{CO}}-\right)$, $69.38(\mathrm{~d},-\mathrm{CHOH}), 41.70(\mathrm{t}), 38.11(\mathrm{t}), 32.94(\mathrm{t}), 30.33(\mathrm{t})$, $26.55(\mathrm{t}), 23.63(\mathrm{t}), 14.42\left(\mathrm{q},-\underline{-} \mathrm{CH}_{3}\right)$.

\section{(S)-N,3-dihydroxynonanamide (S-11)}

Hydroxamic acid $\boldsymbol{S - 1 1}$ was prepared in the same manner as its enantiomer $\boldsymbol{R}-11$. Pure compound ( $>97 \%, \mathrm{GC}$ after derivatization of sample) in form of solid with a slight yellow tint was obtained with $41 \%$ yield. $[\alpha]_{D}^{23}+4.11$ (c $\left.2.65, \mathrm{MeOH}\right), \mathrm{mp}$ 
104.8-108.6 ${ }^{\circ} \mathrm{C}$ (acetone). Its IR, MS, and NMR spectra matched those determined for compound $\boldsymbol{R}-\mathbf{1 1}$.

\section{(R)-3-hydroxy- $N$-methylnonanamide $(R-12)$}

A solution of potassium hydroxide $(1.3 \mathrm{~g}, 0.023 \mathrm{~mol})$ in methanol $(5 \mathrm{~mL})$ was added to a solution of methylamine hydrochloride $(1.1 \mathrm{~g}, 0.016 \mathrm{~mol})$ in methanol $(10 \mathrm{~mL})$ at $0{ }^{\circ} \mathrm{C}$. The mixture was stirred in an ice bath for $15 \mathrm{~min}$ and then the precipitated potassium chloride was filtered off. The filtrate was added to methyl ester of $(R)$-3-hydroxynonanoic acid $(\boldsymbol{R}-6 ; 1.5 \mathrm{~g}, 0.008 \mathrm{~mol})$ and after thorough mixing kept at room temperature for $24 \mathrm{~h}$. Then, Dowex $50 \mathrm{~W}-\mathrm{X} 8$ resin was added to obtain a $\mathrm{pH}$ of 6 and the solution was filtered. The solvent was evaporated and the crude product was purified by crystallization using acetone. Pure (>99\%, GC) amide $\boldsymbol{R}-\mathbf{1 2}(0.463 \mathrm{~g})$ was obtained with $31 \%$ yield. $[\alpha]_{D}^{23}-25.03$ (c $5, \mathrm{CHCl}_{3}$ ), mp $78.5-80.4{ }^{\circ} \mathrm{C}$ (acetone). IR $\left(\mathrm{cm}^{-1}\right.$, neat): 3289.7, 3092, 2925.2, 2846.1, 1640.5, 1559.8, 1402.1, 1071.7, 861.4. GC-MS (EI, $70 \mathrm{eV})$, $m / z: 186\left(\mathrm{M}^{+}-1,1\right), 102$ (100), 73 (56), 69 (8), 58 (63), 55 (23), 45 (32), 43 (33), 41 (23), 39 (6). ${ }^{1} \mathrm{H}$ NMR ( $\delta$ ppm): 6.18 (br.s, 1H; -NH-), 3.95 (m, 1H; -CㅂH), 3.74 (br.s, 1H; $-\mathrm{OH}$ ), 2.79 (d $\left.J=4.8 \mathrm{~Hz}, 3 \mathrm{H} ;-\mathrm{NHCH}_{3}\right), 2.35$ (dd $J=$ $15.3 \mathrm{~Hz}, J=3 \mathrm{~Hz}, 1 \mathrm{H}$; $\left.-\mathrm{CHCH}_{2} \mathrm{CO}-\right), \overline{2.22}$ (dd $J=15.3 \mathrm{~Hz}$, $J=8.8 \mathrm{~Hz}, 1 \mathrm{H} ;-\mathrm{CHCH}_{2} \mathrm{CO}-$ ), 1.40 (br.m, $10 \mathrm{H}$ ), 0.85 (t $J$ $\left.=6.6 \mathrm{~Hz} ;-\mathrm{CH}_{3}\right) .{ }^{13} \mathrm{C}$ NMR: $173.24(\mathrm{~s} ;-\underline{\mathrm{CO}}-), 68.65(\mathrm{~d}$; - $\underline{\mathrm{CHOH}}$, 42.29 (t), $36.91(\mathrm{t}), 31.71(\mathrm{t}), 29.15$ (t), 26.06 (q; $\left.-\mathrm{NHC}_{3}\right), 25.40(\mathrm{t}), 22.52(\mathrm{t}), 14.01\left(\mathrm{q} ;-\underline{-} \mathrm{CH}_{3}\right)$.

\section{(S)-3-hydroxy-N-methylnonanamide (S-12)}

Compound $S \mathbf{- 1 2}$ was obtained according to the method described for amide $\boldsymbol{R} \mathbf{- 1 2}$. Crystallization in acetone gave pure (>99\%, GC) amide $\boldsymbol{S - 1 2}(0.465 \mathrm{~g})$ with $31 \%$ yield. $[\alpha]_{D}^{23}+24.83$ (c 5, $\mathrm{CHCl}_{3}$ ), mp $81.1-82.9^{\circ} \mathrm{C}$ (acetone). Its IR, MS, and NMR spectra matched those determined for compound $\boldsymbol{R} \mathbf{- 1 2}$.

\section{Results and discussion}

Preparation of corresponding starting materials, i.e., enantiomeric forms of methyl esters of RA and 3-hydroxamic acid was the first step (Scheme 1). Methyl $(R)$-ricinoleate $(\boldsymbol{R}-\mathbf{1})$ was obtained from castor oil by common transesterification with methanol. It was then used as a substrate in the three-step process of inversion leading to its $S$ enantiomer (S-1) (Kula et al. 2014). In order to obtain 9-carbon skeleton derivatives of RA we performed ozonolysis process similarly as reported elsewhere (Kula et al. 2000). Castor oil and methyl $(S)$-ricinoleate were used as substrates for the production of dimethyl acetals of $(R)$ - and $(S)$-3hydroxynonanal $(\boldsymbol{R}-\mathbf{5}$ and $\boldsymbol{S} \mathbf{- 5})$, respectively. Then, the obtained compounds $\boldsymbol{R} \mathbf{- 5}$ and $\mathbf{S} \mathbf{- 5}$ were transformed into methyl esters of $(R)$ - and $(S)$-3-hydroxynonanoic acid $(\boldsymbol{R}-\mathbf{6}$, $\boldsymbol{S}$-6) by oxidation with hydrogen peroxide and hydrochloric acid in methanol with about $50 \%$ yield. The oxidation was performed according to the published method (Takeda et al. 1997), however, some modifications of this procedure were made. Preparation of the methyl esters of $(R)$-3-hydroxynonanoic acid (R-6) by ozonolysis of castor oil was previously described in literature, however, in all cases the product of that reaction was a mixture of compounds (Ishmuratov et al. 2006, 2007, 2009).

Consequently, a series of amides and hydroxamic acids derived from ricinoleic and 3-hydroxynonanoic acids were prepared. Starting from the methyl esters of RA $(\boldsymbol{R}-\mathbf{1}, \boldsymbol{S}-\mathbf{1}) 6$ compounds (i.e., 3 pairs of enantiomers), among them two primary amides $(\boldsymbol{R}-\mathbf{2}, \boldsymbol{S} \mathbf{- 2})$, two hydroxamic acids $(\boldsymbol{R}-\mathbf{3}, \boldsymbol{S}$ 3), and two $N$-methyl amides $(\boldsymbol{R}-\mathbf{4}, \boldsymbol{S}$-4) were synthesized (Scheme 2).

On the other hand, the methyl esters of $(R)$ - and $(S)-3$ hydroxynonanoic acid $(\boldsymbol{R}-\mathbf{6}, \boldsymbol{S}-\mathbf{6})$ were used for the preparation of 12 compounds (6 pairs of enantiomers). Like in the case of RA, two primary amides $(\boldsymbol{R}-7, S-7)$, two hydroxamic acids $(\boldsymbol{R}-\mathbf{1 1}, \boldsymbol{S}$-11), and two $N$-methyl amides $(\boldsymbol{R}-12, \boldsymbol{S}$-12) were obtained from these 9-carbon skeleton

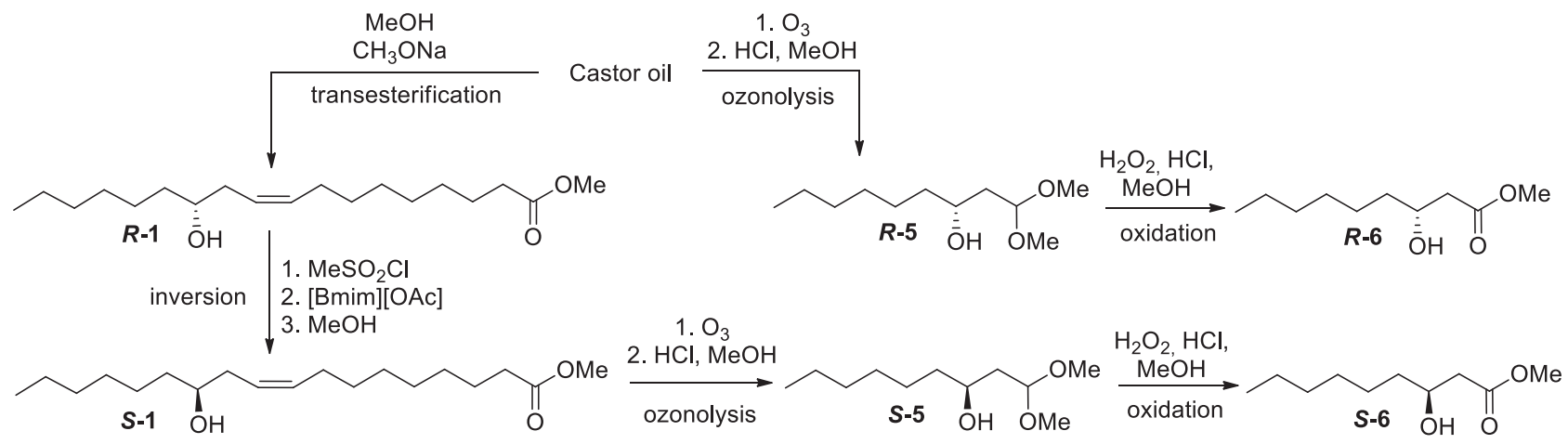

Scheme 1 Synthesis of methyl esters of ricinoleic acid $(\boldsymbol{R}-\mathbf{1}, \boldsymbol{S}-\mathbf{1})$, dimethyl acetals of 3-hydroxynonanal $(\boldsymbol{R}-\mathbf{5}, \boldsymbol{S}-\mathbf{5})$, and methyl esters of 3-hydroxynonanoic acid $(\boldsymbol{R}-\mathbf{6}, \boldsymbol{S}-\mathbf{6})$ 


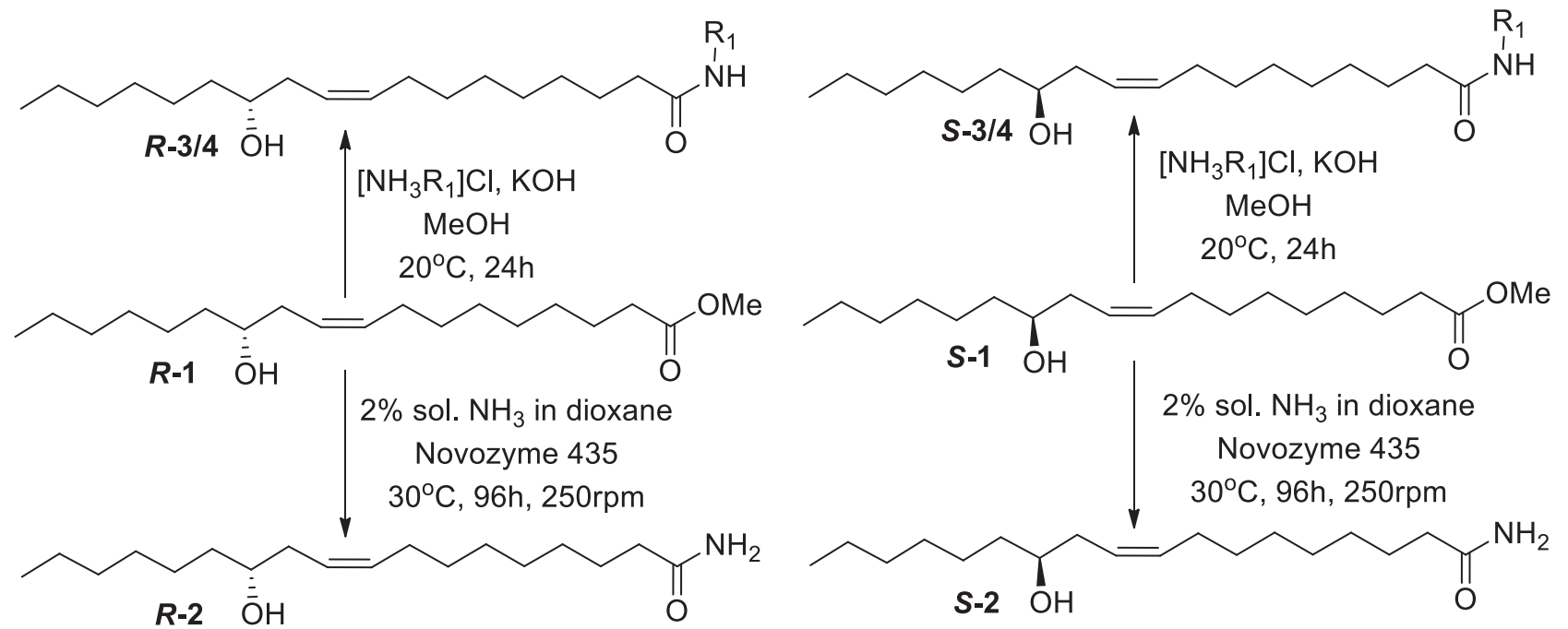

Scheme 2 Synthesis of $(R)$ - and $(S)$-ricinoleic acid derivatives: primary amides $\boldsymbol{R}-\mathbf{2}$ and $\boldsymbol{S}$-2, hydroxamic acids $\boldsymbol{R}-\mathbf{3}$ and $\boldsymbol{S}-\mathbf{3}\left(\mathrm{R}_{1}=\mathrm{OH}\right)$, and $N$-methyl amides $\boldsymbol{R}-\mathbf{4}$ and $\boldsymbol{S}-\mathbf{4}\left(\mathrm{R}_{1}=\mathrm{CH}_{3}\right)$

Scheme 3 Synthesis of $(R)$ - and (S)-3-hydroxynonanoic acid derivatives: primary amides $(\boldsymbol{R}$ 7, $S$-7), ethanolamine amides $(\boldsymbol{R}-8, S-8)$, 2-amino-2-methyl-1pyrrolidine amides $(\boldsymbol{R}-\mathbf{1 0}, \boldsymbol{S}-\mathbf{1 0})$, hydroxamic acids $(\boldsymbol{R}-\mathbf{1 1}, \boldsymbol{S}-\mathbf{1 1}$; $\mathrm{R}_{4}=\mathrm{OH}$ ), and $N$-methyl amides $\left(\boldsymbol{R}-12, \boldsymbol{S}-12 ; \mathrm{R}_{4}=\mathrm{CH}_{3}\right)$ propanol amides $(\boldsymbol{R}-\mathbf{9}, \boldsymbol{S}-\mathbf{9})$,

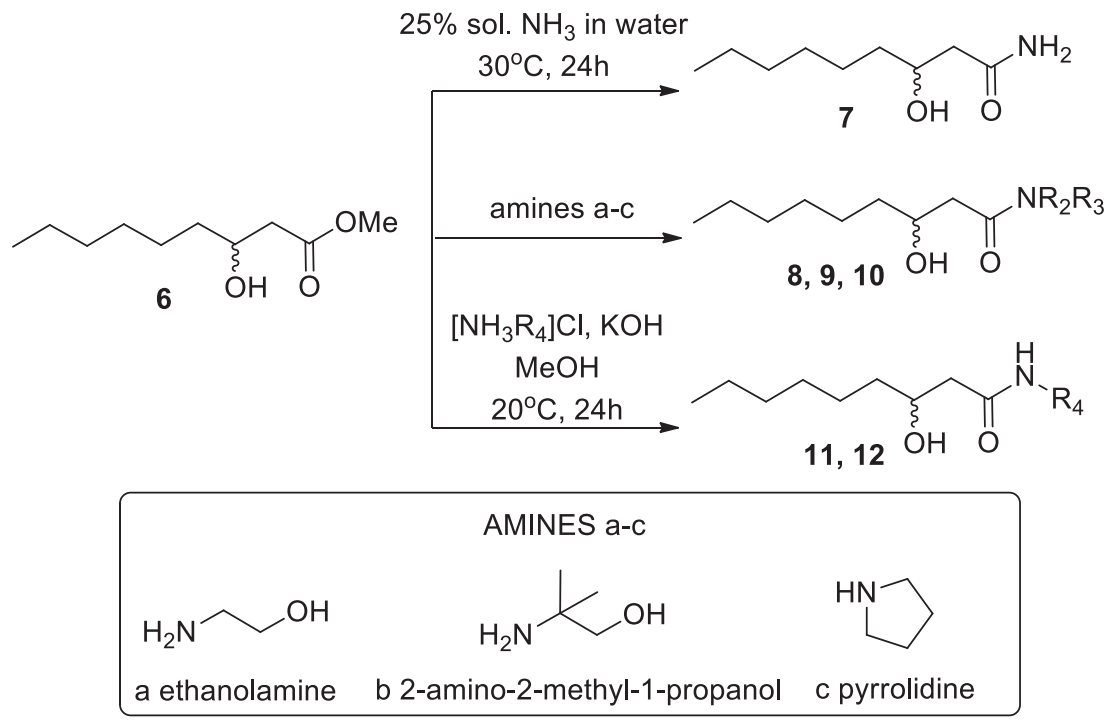

fatty acids. Moreover, six other enantiomeric amides of 3-hydroxynonanoic acids, i.e., with ethanolamine ( $R-8, S-8)$, 2-amino-2-methyl-1-propanol (R-9, S-9) and pyrrolidine $(\boldsymbol{R}-10, \boldsymbol{S}-10)$ were also synthesized (Scheme 3 ). We decided to prepare and investigate these six chiral compounds because of good anticancer and antimicrobial activity of analogical amides, especially for derivatives with ethanolamine and pyrrolidine derived from $(R)$ - and $(S)$-RA and reported earlier (Matysiak et al. 2017, 2018). Moreover, impact of the stereogenic center on their biological activity was observed.

Hydroxamic acids and $N$-methyl amides were synthesized basing on the previously published methods (Hauser and Renfrow 1939; Devlin et al. 1975). Reaction of methyl esters of RA and 3-hydroxynonanoic acid with hydroxylamine or methylamine was performed in the presence of $\mathrm{KOH}$ in methanol at room temperature for $24 \mathrm{~h}$. These reactions proceeded with good yields, ranging from 31 to $62 \%$.

Primary amides derived from RA $(\boldsymbol{R}-\mathbf{2}, \boldsymbol{S}-\mathbf{2})$ were synthesized using the enzymatic method catalyzed by Novozyme 435 (lipase from Candida antarctica) and performed in $2 \%$ dioxane solution of $\mathrm{NH}_{3}$ by mixing all substrates at $30{ }^{\circ} \mathrm{C}$ (García et al. 1994). The reaction yield was good ranging from 46 to $52 \%$. In the case of primary amides of 3-hydroxynonanoic acid ( $\boldsymbol{R}-\mathbf{7}$ and $\boldsymbol{S}-\mathbf{7})$, another, very simple and fast method was used. Good yields (50-58\%) were obtained when starting the methyl esters ( $\boldsymbol{R}-\mathbf{6}$ and $\boldsymbol{S}$-6) were 
shaken with $25 \%$ water solution of $\mathrm{NH}_{3}$ at $30{ }^{\circ} \mathrm{C}$ without presence of the enzyme.

The amides $(\mathbf{8}-\mathbf{1 0})$ were prepared by a simple reaction of methyl esters of 3-hydroxynonanoic acid with corresponding amines, i.e., ethanolamine, 2-amino-2-methyl-1-propanol, and pyrrolidine. The reactions were carried out by heating the substrates at the proper temperature (keeping a gentle reflux) without a solvent. This kind of method for preparation of amides was previously used to synthesize analog derivatives of $(R)$ - and $(S)$-ricinoleic acids (Matysiak et al. 2017, 2018). It was not only environment-friendly and free of the solvent procedure, but it also provided relatively good performance. The amides (8-10) synthesized in the present study were obtained in good yields, ranging from $39 \%$ to $95 \%$. The best yields (87-95\%) were observed for the derivatives with 2-amino-2-methyl-1-propanol ( $\boldsymbol{R}-\mathbf{9}$ and $S$-9). It was surprising, because in our previous study in the case of analogical amides of $(R)$ - and $(S)$-ricinoleic acids we observed significantly weaker results ranging from 43 to 48\% (Matysiak et al. 2018).

In general, we obtained 24 chiral compounds eight of which are derivatives of RA (1-4), two dimethyl acetals of 3-hydroxynonanal (5) and 14 derivatives of 3hydroxynonanoic acid (6-12). It is worth mentioning that only the starting methyl esters of RA $(\boldsymbol{R}-\mathbf{1}, \mathrm{S}-\mathbf{1})$ and the methyl ester of $(R)$-3-hydroxynonanoic acid $(\boldsymbol{R}-\mathbf{6})$ have been fully spectrally and polarimetrically characterized so far (Odham and Samuelsen 1970; Ishmuratov et al. 2006, 2007, 2009; Jiang et al. 2010; Kula et al. 2014). Moreover, the compounds $\boldsymbol{R}-\mathbf{1}$ and $\boldsymbol{S}-\mathbf{1}$ were studied with regard to biological activity. Some information about the compounds $\boldsymbol{R}-\mathbf{2}$ and $\boldsymbol{R}-\mathbf{4}$ which are amides of $(R)$-RA can also be found (Galstukhova 1960; Applewhite et al. 1963). The methods for their preparation and their melting points were described, but their specific optical rotations were not given. Applewhite et al. (1963) described the melting point of the compound $\boldsymbol{R}-\mathbf{4}\left(30.5-31.5^{\circ} \mathrm{C}\right)$, however, we were not able to determine this parameter during our research due to the semisolid consistency of this derivative. The other obtained compounds have been neither synthesized nor described in literature, and all the more tested for cytotoxic activity. In general, in the group of 24 compounds derived from ricinoleic and 3-hydroxynonanoic acids, including methyl esters, hydroxamic acids, and amides, 16 of them were new compounds. Therefore, we carried out full spectral and polarimetric characterization of all the obtained RA derivatives. The purity of all compounds was monitored by TLC, ${ }^{13} \mathrm{C}$ NMR, GC, and measurements of their optical rotations. What is more, ${ }^{1} \mathrm{H}$ NMR, ${ }^{13} \mathrm{C}$ NMR, GC-MS, and IR analyses were done for confirmation of their structures.

Then, the effects of the obtained compounds on cancer cell growth and proliferation were determined by a colorimetric MTT assay. Two cancer cell lines-HT29 (human colorectal adenocarcinoma) and HeLa (human cervical adenocarcinoma) — were used in the experiments. The cells were treated with various concentrations of the compounds for $72 \mathrm{~h}$ and the cytotoxicity was expressed as the $\mathrm{IC}_{50}$ value (concentration of the tested compound that reduces the absorbance by $50 \%$ when compared to the negative control). The results of the cytotoxicity assay are presented in Table 1.

Cytotoxic activities of the tested compounds expressed as $\mathrm{IC}_{50}$ values ranged from 13.22 to $80.00 \mu \mathrm{M}$. The results also showed that there was no significant difference between the activities of $(R)$ - and $(S)$-enantiomers of particular derivatives. For the majority of tested compounds higher toxicity was observed against HT29 than HeLa cancer cells. Derivatives 3 and 11, i.e., hydroxamic acids were exceptions to this rule. Toxicity of the compound $\mathbf{3}$ was similar both against HT29 and HeLa cells $\left(\right.$ IC $_{50}$ from 41.11 to $44.03 \mu \mathrm{M}$; Table 1). The most interesting results were obtained for the hydroxamic acids derived from 3hydroxynonanoic acid (11), which were found to be the most cytotoxic for HeLa cancer cells ( $\mathrm{IC}_{50}$ values: $13.22 \mu \mathrm{M}$
Table 1 The $\mathrm{IC}_{50}$ values $(\mu \mathrm{M})$ determined after 72 -h treatment of cancer cells with the tested compounds

\begin{tabular}{llllll}
\hline Compound & \multicolumn{3}{l}{ HT29 } & & HeLa \\
\cline { 2 - 3 } \cline { 5 - 6 } & $(R)$-enantiomer & $(S)$-enantiomer & & $(R)$-enantiomer & $(S)$-enantiomer \\
\hline 2 & $41.25 \pm 2.72$ & $42.78 \pm 4.20$ & & $60.56 \pm 4.40$ & $57.00 \pm 3.12$ \\
3 & $44.03 \pm 3.23$ & $41.11 \pm 2.02$ & & $41.56 \pm 2.83$ & $44.00 \pm 2.34$ \\
4 & $40.42 \pm 2.37$ & $42.92 \pm 1.72$ & & $62.67 \pm 2.09$ & $56.89 \pm 1.44$ \\
6 & $43.75 \pm 2.51$ & $43.75 \pm 2.28$ & & $70.56 \pm 5.09$ & $77.00 \pm 4.96$ \\
7 & $76.25 \pm 3.35$ & $64.50 \pm 2.80$ & & $79.33 \pm 5.70$ & $80.00 \pm 3.21$ \\
8 & $45.42 \pm 2.07$ & $47.08 \pm 3.33$ & & $73.67 \pm 4.97$ & $63.89 \pm 1.96$ \\
9 & $43.33 \pm 1.49$ & $49.17 \pm 2.25$ & & $64.00 \pm 3.46$ & $66.00 \pm 2.27$ \\
10 & $52.78 \pm 2.89$ & $52.50 \pm 3.06$ & & $54.89 \pm 6.42$ & $58.67 \pm 3.79$ \\
11 & $41.42 \pm 2.16$ & $42.42 \pm 2.15$ & & $13.22 \pm 1.25$ & $14.33 \pm 1.05$ \\
12 & $50.28 \pm 1.73$ & $53.19 \pm 2.40$ & & $54.00 \pm 2.65$ & $57.67 \pm 4.64$ \\
\hline
\end{tabular}


and 14.33 for $(R)$ - and (S)-enantiomer, respectively) while HT29 cells were more resistant to it (IC 50 values: $41.42 \mu \mathrm{M}$ and 42.42 for $(R)$ - and $(S)$-enantiomer, respectively).

Comparing these results with our previous findings (Matysiak et al. 2017) we can observe that modification of the parent methyl ricinoleate with amines resulted in increased cytotoxicity. In the case of HT29 cells, methyl esters of RA showed weak activity ( $\mathrm{IC}_{50}$ values: 164.8-175.3 $\mu \mathrm{M}$ ), whereas, the amides (2-4) obtained in this study were significantly more toxic against the tested cancer cells with $\mathrm{IC}_{50}$ values ranging from 40.42 to $44.03 \mu \mathrm{M}$.

Interestingly, such a relationship was not observed in the case of 3-hydroxynonanoic acid derivatives (6-12) and their activities varied depending on cancer cell line. In the case of HT29 cells, the starting methyl 3-hydroxynonanoates (6; $\mathrm{IC}_{50}$ value $43.75 \mu \mathrm{M}$ ) showed similar cytotoxicity as their amide derivatives $\mathbf{8 ,} \mathbf{9}, \mathbf{1 1}\left(\mathrm{IC}_{50}\right.$ values from 41.42 to $47.08 \mu \mathrm{M}$ ), and even better activity than compounds $\mathbf{7 , 1 0}$, and 12 ( $\mathrm{IC}_{50}$ values: $50.28-76.25 \mu \mathrm{M}$ ). In the case of HeLa cancer cells, the starting methyl esters 6 and amides 7, 8, 9 exhibited similar activity with $\mathrm{IC}_{50}$ values in the range from 64.00 to $80.00 \mu \mathrm{M}$. Slightly greater cytotoxicity was observed for the compounds $\mathbf{1 0}$ and $\mathbf{1 2}\left(\mathrm{IC}_{50}\right.$ values $54.00-58.67 \mu \mathrm{M}$ ), and the derivatives $\mathbf{1 1}$ were significantly more active against the tested cells $\left(\mathrm{IC}_{50}\right.$ values: $13.22 \mu \mathrm{M}$ and 14.33 for $(R)$ - and (S)-enantiomer, respectively) as compared to the starting compounds. Therefore, it can be observed that the modification of the parent methyl esters of 3-hydroxynonanoic acid with amines does not increase cytotoxicity. In general, in the case of 9-carbon skeleton derivatives the least toxicity against HT29 and HeLa cancer cells was observed for the primary amides of 3hydroxynonanoic acid $\mathbf{7}$ and the greatest activity for the compounds 11, i.e., hydroxamic acids.

\section{Conclusion}

Two series of 24 chiral hydroxy acid derivatives containing 18 and 9 carbon skeletons were synthesized starting from castor oil in satisfying yields using chemical and biochemical methods. Sixteen of the amide hydroxy acids, including two hyrdoxamic acids, were obtained and characterized spectrally and polarymetrically for the first time.

All the compounds showed cytotoxicity against HT29 and HeLa cancer cells, but the results were varied. Most of the tested ricinoleic and 3-hydroxynonanoic acids derivatives were more toxic against HT29 than HeLa cancer cells. However, the strongest cytotoxic effect was observed for compounds 11, i.e., hydroxamic acids derived from 3hydroxynonanoic acid, against $\mathrm{HeLa}$ cells. The $\mathrm{IC}_{50}$ values for these derivatives ranged from $13.22 \mu \mathrm{M}$ to 14.33 for $(R)$ and $(S)$-enantiomer, respectively, and they can be considered as compounds of potential pharmacological significance. The results also showed that there was no significant difference between activities of $(R)$ - and $(S)$ enantiomers of particular derivatives.

Acknowledgements The authors want to thank Prof. Krzysztof Śmigielski for his help in ozonolysis process.

\section{Compliance with ethical standards}

Conflict of interest The authors declare that they have no conflict of interest.

Publisher's note: Springer Nature remains neutral with regard to jurisdictional claims in published maps and institutional affiliations.

Open Access This article is distributed under the terms of the Creative Commons Attribution 4.0 International License (http://crea tivecommons.org/licenses/by/4.0/), which permits use, duplication, adaptation, distribution, and reproduction in any medium or format, as long as you give appropriate credit to the original author(s) and the source, provide a link to the Creative Commons license, and indicate if changes were made.

\section{References}

Applewhite TH, Nelson JS, Goldblatt LA (1963) Castor-based derivatives: synthesis of some amides. J Am Oil Chem Soc 40:101-104

D'Oca CDRM, Coelho T, Marinho TG, Hack CRL, Duarte RC, da Silva PA, D'Oca MGM (2010) Synthesis and antituberculosis activity of new fatty acid amides. Bioorg Med Chem Lett 20:5255-5257

Devlin JP, Ollis WD, Thorpe JE, Wright DE (1975) Studies concerning the antibiotic actinonin. Part VI. Synthesis of structural analogues of actinonin by dicyclohexylcarbodi-imide coupling reactions. J Chem Soc Perkin Trans 1 9:848-851

Dos Santos DS, Piovesan LA, D'Oca CRM, Hack CRL, Treptow TGM, Rodrigues MO, Vendramini-Costa DB, Ruiz ALTG, de Carvalho JE, D'Oca MGM (2015) Antiproliferative activity of synthetic fatty acid amides from renewable resources. Bioorg Med Chem 23:340-347

Farrell EK, Merkler DJ (2008) Biosynthesis, degradation and pharmacological importance of the fatty acid amides. Drug Discov Today 13:558-568

Galstukhova N (1960) Some reactions of ricinoleic acid and its derivatives. Russ J Gen Chem 30:1387-1389

García MJ, Rebolledo F, Gotor V (1994) Lipase-catalyzed aminolysis and ammonolysis of $\beta$-ketoesters. Synthesis of optically active $\beta$ ketoamides. Tetrahedron 50:6935-6940

Hauser CR, Renfrow WBJ (1939) Benzohydroxamic acid. Org Synth 19:15

Ishmuratov GY, Legostaeva YV, Botsman LP, Yakovleva MP, Shakhanova OO, Muslukhov RR, Tolstikov GA (2009) Transformations of peroxide ozonolysis products of natural olefins by $\mathrm{N}$-containing organic compounds in methanol. Chem Nat Compd 45:318-321

Ishmuratov GY, Shayakhmetova AK, Yakovleva MP, Legostaeva YV, Shitikova OV, Galkin EG, Tolstikov GA (2007) Ozonolysis of alkenes and study of reactions of polyfunctional compounds: LXVIII. Investigation of transformations of peroxide products of olefins ozonolysis treated with hydroxylamine hydrochloride. Russ J Org Chem 43:1114-1119

Ishmuratov GY, Yakovleva MP, Shayakhmetova AK, Botsman LP, Nasibullina GV, Muslukhov RR, Tolstikov GA (2006) 
Ozonolytic transformations of olefinic derivatives of L-menthol and ricinolic acid. Chem Nat Compd 42:631-635

Jiang H, Gschwend B, Albrecht $Ł$, Anker Jørgensen K (2010) Organocatalytic preparation of simple $\beta$-hydroxy and $\beta$-amino esters: low catalyst loadings and gram-scale synthesis. Org Lett 12:5052-5055

Kula J (1999) Safer ozonolysis reactions: a compilation of laboratory experience. Chem Health Saf 6:21-22

Kula J, Bonikowski R, Szewczyk M, Ciolak K (2014) Synthesis of $(S)$-ricinoleic acid and its methyl ester with the participation of ionic liquid. Chem Phys Lipids 183:137-141

Kula J, Masarweh A (1998) 12-nor-alloisolongifolan-11-one, a ketone with amber-like odour from longifolene. Flavour Fragr J 13:277-278

Kula J, Quang TB, Sikora M (2000) Synthesis of enantiomerically pure volatile compounds derived from $(R)$-3-hydroxynonanal. Tetrahedron Asymmetry 11:943-950

Kuppala R, Govindarajan M, Tambat R, Patel N, Nandanwar H, Bhutani KK, Kartha KPR (2016) Synthesis and antibacterial activity of ricinoleic acid glycosides. RSC Adv 6:3700-3713

Matysiak S, Chmiel A, Skolimowski J, Kula J, Pasternak B, Blaszczyk A (2017) Synthesis and cytotoxicity of $(R)$ - and $(S)$-ricinoleic acid amides and their acetates. Chirality 29:616-622
Matysiak S, Zabielska J, Kula J, Kunicka-Styczyńska A (2018) Synthesis of $(R)$ - and $(S)$-ricinoleic acid amides and evaluation of their antimicrobial activity. J Am Oil Chem Soc 95:69-77

Montalbetti CAGN, Falque V (2005) Amide bond formation and peptide coupling. Tetrahedron 61:10827-10852

Narasimhan B, Mourya VK, Dhake AS (2007) QSAR studies of antibacterial ricinoleic acid derivatives. Pharm Chem J 41:133-139

Odham G, Samuelsen B (1970) On the methylation and demethylation of optically active aliphatic hydroxy compounds. Acta Chem Scand 24:468-472

Pabiś S, Kula J (2016) Synthesis and bioactivity of $(R)$-ricinoleic acid derivatives: a review. Curr Med Chem 23:4037-4056

Pinto DCGA, Silva AMS (2016) Hydroxamic acids, recent breakthroughs in stereoselective synthesis and biological evaluations. Curr Org Synth 13:659-668

Salywon AM, Dierig DA, Rebman JP, De Rodríguez DJ (2005) Evaluation of new Lesquerella and Physaria (Brassicaceae) oilseed germplasm. Am J Bot 92:53-62

Takeda T, Watanabe H, Kitahara T (1997) Direct oxidation of acetals and aldehydes to esters. Synlett 1997:1149-1150 